SCIENTIFIC REPORT

\title{
MMP inhibition prevents human lens epithelial cell migration and contraction of the lens capsule
}

\author{
T T L Wong, J T Daniels, J G Crowston, P T Khaw
}

Br J Ophthalmol 2004;88:868-872. doi: 10.1136/bjo.2003.034629

See end of article for authors' affiliations

a.t.

Correspondence to: DrT T L Wong, Ocular Repair and Regeneration Biology, Department of Pathology, Institute of Ophthalmology, 11-43 Bath Street, London ECIV 9EL, UK; tina.wong@udl. ac.uk

Accepted

30 November 2003
Purpose: The development of posterior capsule contraction following cataract surgery is caused by the activity of residual lens epithelial cells. Matrix metalloproteinases (MMPs) are a group of proteolytic enzymes, which are essential for cell migration and cell mediated contraction following wound healing. The authors investigated whether inhibiting MMP activity can reduce lens epithelial cell migration and as a result, lead to a reduction in cell mediated capsule contraction.

Methods: Human donor lens capsules were cultured and treated with a broad spectrum MMP inhibitor, llomastat (GM6001). MMP-2 and MMP-9 production were determined by ELISA. Cell migration onto the posterior capsule and capsule contraction were digitally measured.

Results: MMP inhibition significantly reduced lens epithelial cell migration onto the posterior capsule $(p<0.05)$, and a reduction in capsule contraction was observed $(p<0.05)$.

Conclusions: Ilomastat significantly reduced lens epithelial cell migration onto the posterior capsule surface and inhibited capsule contraction. MMP inhibition may have a role in the therapeutic treatment of posterior capsule opacification.
C ataract is the most common pathology of the lens. It remains the leading cause of reversible blindness worldwide. Continual improvements in surgical technique and lens implant design have allowed the surgeon to improve on safety and visual outcome. ${ }^{12}$ Posterior capsule opacification (PCO) is the major cause of secondary visual loss following cataract surgery. ${ }^{3}$ A dramatic reduction in reported PCO rates from up to 50\% following surgery down to as low as $7 \%$ have been due in part to advances in intraocular lens design over the last 20 years. ${ }^{45}$

The proliferation and migration of residual lens epithelial cells (LECs) at the equator and under the anterior lens capsule following cataract surgery leads to the development of PCO. ${ }^{6-8}$ Repopulation of the posterior capsule by migrating LECs leading to capsule opacification with associated capsule wrinkling, contraction, and matrix production can result in significant visual impairment.

Matrix metalloproteinases (MMPs) are a group of proteolytic enzymes which are important in many physiological processes such as embryogenesis, development, and wound healing. ${ }^{9}$ Dysregulated MMP activity has long been implicated in diseases associated with uncontrolled proteolysis of connective tissue matrices such as arthritis, ${ }^{10}$ tumourgenesis, ${ }_{11}^{11}$ and tissue ulceration. ${ }^{12}$ MMPs have been found in normal ocular tissues ${ }^{13}$ with overexpression associated with excessive scarring. ${ }^{14}$ The use of synthetic MMP inhibitors in ocular disorders has been shown to reduce tissue damage. ${ }^{15} 16$ Our laboratory has recently shown that these agents inhibit a variety of cell mediated functions such as collagen synthesis, extracellular matrix contraction, and cell migration in Tenon's capsule fibroblasts in vitro. ${ }^{17}$

The aim of this study was to investigate the effect of the synthetic MMP inhibitor, Ilomastat (GM6001) on human lens epithelial cell migration and lens capsule contraction, in an in vitro model of PCO.

\section{METHODS AND MATERIALS}

\section{Retrieval and preparation of human lens capsules}

Donated human eyes were obtained from Moorfields Eye Bank (London, UK) and the Keratec Eye Bank (London, UK).
The research followed the tenets of the Declaration of Helsinki regarding the use of human material. The mean (standard deviation) ages of the donors were 72 (SD 8) years for the female donors and 69 years (SD 7) for the males. All lenses used in the study (total 16) were retrieved within 48 hours postmortem. Lens capsule bag culture was established using a technique previously described. ${ }^{18}$ Briefly, a sham cataract operation was performed, which involved anterior capsulorhexis, nuclear hydroexpression with phosphate buffered saline (PBS), and the aspiration of cortical lens fibres. The dissected lens capsule was transferred into a chamber of a 24 well culture plate. The lens capsule was cultured in $1 \mathrm{ml}$ gelatinase free culture medium, which was composed of Dulbecco's modified Eagle's medium (DMEM) with penicillin ( $100 \mathrm{U} / \mathrm{ml})$, streptomycin $(100 \mu \mathrm{g} / \mathrm{ml})$, gentamicin $(50 \mu \mathrm{g} / \mathrm{ml}), 2 \mathrm{mM}$ L-glutamine, amphoteracin $(0.25 \mu \mathrm{g} / \mathrm{ml})$, and $10 \%(\mathrm{vol} / \mathrm{vol})$ fetal calf serum (FCS) (all Gibco Life Technologies, Paisley, UK). FCS was mixed with gelatin sepharose beads (Amersham Pharmaceutical) in a 1:9 ratio for 1 hour and then filtered to ensure that the serum was gelatinase free. The specimens were incubated at $37^{\circ} \mathrm{C}$ in $5 \%$ humidified $\mathrm{CO}_{2}$ in air.

\section{MMP inhibition}

The effect of Ilomastat on cell migration and capsule contraction was investigated. The inhibitor was prepared as a $10 \mathrm{mM}$ stock solution in dimethyl sulphoxide and test concentrations of $1 \mu \mathrm{M}, 10 \mu \mathrm{M}$, and $100 \mu \mathrm{M}$ were prepared by diluting with gelatinase free $10 \%$ serum containing culture medium. The negative control to Ilomastat (GM6001 negative control; Calbiochem) was also prepared and diluted to a concentration of $100 \mu \mathrm{M}$ with culture medium. A pilot study had previously shown the presence of lens epithelial cells at the equator by day 3 of culture in $10 \%$ serum. Based on this observation, a study was designed and repeated four times, whereby the serum containing culture medium was replaced

Abbreviations: DMEM, Dulbecco's modified Eagle's medium; FCS, fetal calf serum; LECs, lens epithelial cells; MMP, matrix metalloproteinase; PBS, phosphate buffered saline; PCO, posterior capsule opacification. 
with one of the four test media on day 3 and this marked the start of the study (day 0 ). The test media were changed daily.

\section{MMP protein production}

The culture media bathing the lens capsules were collected on days 5, 10, and 15; stored at $-70^{\circ} \mathrm{C}$ then lyophilised and reconstituted in PBS before use. Total MMP-2 and MMP-9 were quantified in the collected culture media taken from the epithelial cell populated lens capsules by enzyme linked immunosorbent assay (ELISA). The ELISA kits were solid phase enzyme immunoassays based on a two step sandwich format and measured both the latent and active forms of the enzymes (Amersham BioSciences, UK).

A test run was performed for each assay to determine the appropriate dilution factor of $100 \mu \mathrm{l}$ of each collected sample culture medium to ensure that the readings correlated with the exponential part of the respective standard curves. To eliminate the possibility of interassay variation for each ELISA, the samples were assayed in one run. The assays were performed in accordance with the manufacturer's instructions.

\section{Lens epithelial cell migration}

Epithelial cell migration onto the posterior capsule was measured by capturing a series of digital images taken on days 5,10 , and 15 . The settings employed to photograph the capsules were standardised by using fixed camera and microscope settings and magnification respectively. The images were taken by attaching the camera lens to a customised microscope eyepiece. The distance travelled by the lens epithelial cells was measured from a predetermined mark drawn on the base of the culture dish at the leading edge of the epithelial cell population on the day the cells started to appear-that is, day 3 in culture, before the study started. The average distance travelled by the lens epithelial cells from the marked position was measured in pixels by using UTHSCSA (University of Texas Health Science Center in San Antonio) Imagetool Software. Measurements from the mark to the leading edge of the migrating cells were taken from 10 points on the mark. Each chosen point on the mark was a fixed distance apart, determined by measurements using the software.

\section{Lens capsule contraction}

Digital images of the lens capsules were captured and the measurements were again standardised as previously described. The vertical and horizontal capsule diameters between the pins were measured using UTHSCSA Imagetool Software, and the average diameter for each lens capsule was calculated from the total sum of the six repeated measurements at each axis.

\section{Cell viability}

To establish that the inhibitory effect of Ilomastat on cell migration was not caused by cell toxicity, cell viability was determined. Lens epithelial cell proliferation in the presence of $1 \mu \mathrm{M}, 10 \mu \mathrm{M}$, and $100 \mu \mathrm{M}$ Ilomastat was determined using a kit incorporating the WST-1 reagent (Boehringer Mannheim Diagnostics and Biochemicals, Lewes, UK) in accordance to the manufacturer's instructions. Briefly, lens capsules were cultured in serum until proliferating lens epithelial cells migrated and formed a confluent monolayer on the culture plate. The cells were then trypsinised and reseeded into 96 well tissue culture plates, at a density of $1 \times 10^{3}$ per well in serum containing culture medium and cultured for 4 hours. The culture medium was removed and the cells were washed three times with PBS before the addition of medium containing concentrations of Ilomastat ( $1 \mu \mathrm{M}, 10 \mu \mathrm{M}, 100 \mu \mathrm{M}$, and negative control). Three plates per assay were seeded and prepared for harvesting on days 5 , 10 , and 15, the same time points used in the study. At each time interval $10 \mu \mathrm{l}$ of WST-1 reagent was added to each well and incubated for 2 hours at $37^{\circ} \mathrm{C}$. The absorbance, which relates to the number of viable cells converting the reagent to coloured formazan crystals, was read at $450 \mathrm{~nm}$. The cells were re-fed daily with the test media.

\section{Statistical evaluation}

Statistical analysis was performed using Excel (Microsoft). Comparisons of data samples from contraction assays were performed by analysis of variance (ANOVA), which allowed multiple comparisons between two or more data sets. For all analyses, $\mathrm{p}<0.05$ was considered statistically significant with a $95 \%$ confidence interval.

\section{RESULTS}

MMP production during lens epithelial cell migration Total MMP-2 and MMP-9 protein production increased with time with maximum levels reached on day 15. MMP levels were reduced with Ilomastat, with the greatest inhibitory effect being seen at the higher concentrations $(10 \mu \mathrm{M}$ and $100 \mu \mathrm{M}, \mathrm{p}<0.05$; fig 1 ). By day 10 a $50 \%$ reduction in total MMP-2 and MMP-9 levels was seen with $10 \mu \mathrm{M}$ and $100 \mu \mathrm{M}$ Ilomastat compared with control $(\mathrm{p}<0.05)$.

Lens epithelial cell migration and capsule contraction Epithelial cell migration onto the posterior capsule was significantly reduced by Ilomastat compared with control $(\mathrm{p}<0.05$; fig 2). On day 5 , a twofold increase in the distance migrated was observed between control and Ilomastat treated capsules at the concentrations $10 \mu \mathrm{M}$ and $100 \mu \mathrm{M}$. By day 10, a fivefold increase in the distance travelled was seen between control and $100 \mu \mathrm{M}$ Ilomastat. In addition, increased capsule wrinkling in the control capsules limited accurate measurement of cell migration.

A confluent monolayer of lens epithelial cells was present in the control capsules at day 10 and evident capsule wrinkling and folds were seen (fig 3A and C). In contrast,
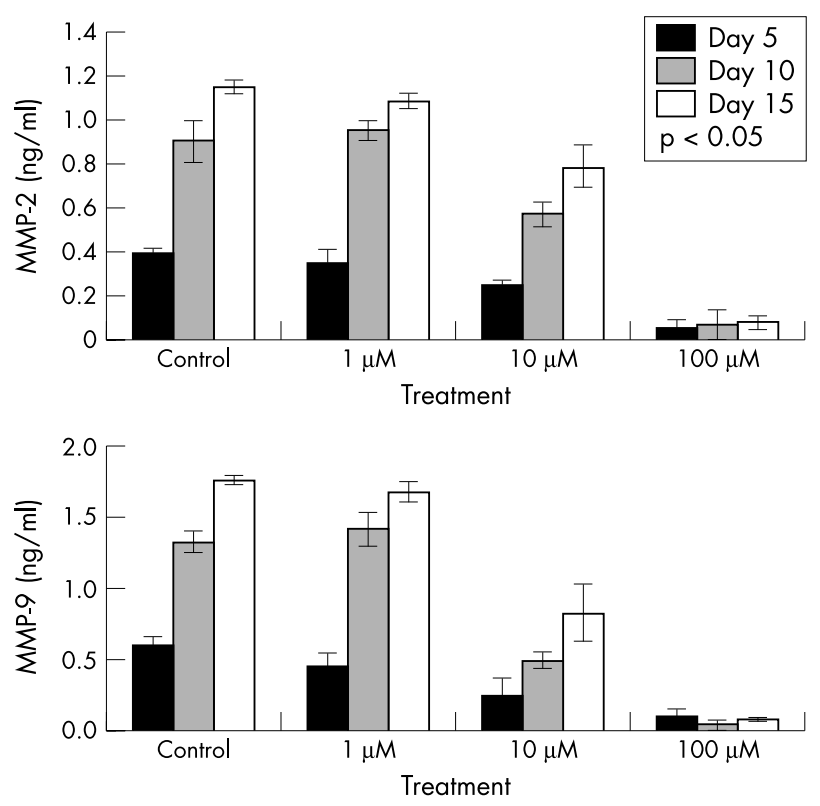

Figure 1 Total MMP-2 and MMP-9 protein production collected in the culture medium was quantified by ELISA. MMP levels were reduced by llomastat in a dose dependent manner compared with control (day 5, day 10 , day $15 ; \mathrm{p}<0.05$ ). 


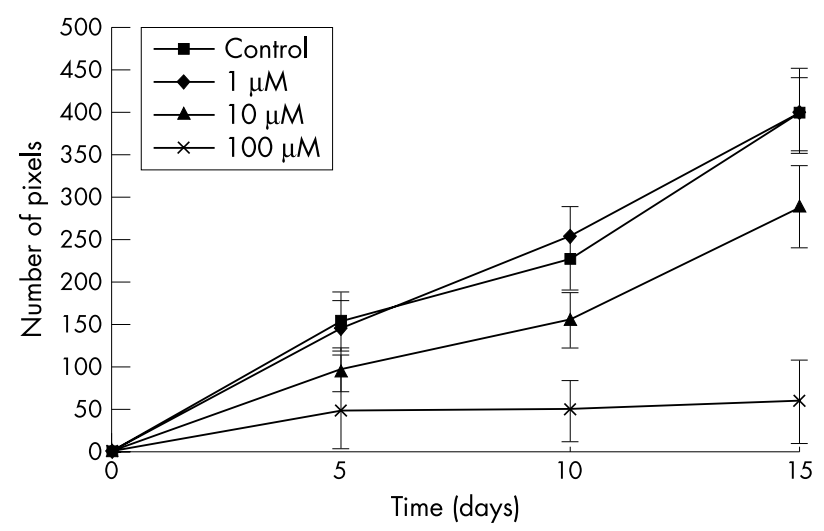

Figure 2 Serial digital photographs were taken of the lens capsules. The capsule diameters between the pins were measured using UTHSCSA (University of Texas Health Science Center in San Antonio) Imagetool Software, and the average diameter for each lens capsule was calculated from six repeated measurements from vertical and horizontal axes. All images were taken at the same marked area at $10 x$ magnification. Three capsules were measured at each treatment group. The graph illustrates the mean distance travelled by the cells and the error bars represent a $95 \%$ confidence interval.

minimal capsule wrinkling was noted with Ilomastat treatment (fig 3B and D). Finally capsule contraction, and thus the reduction of capsule diameter, was dose dependently inhibited by Ilomastat compared with control. Capsule diameter decreased by $60 \%$ in the control treated capsule compared to a $30 \%$ decrease with $10 \mu \mathrm{M}$ and less than $5 \%$ reduction with $100 \mu \mathrm{M}(\mathrm{p}<0.05$, fig 4$)$.

\section{Cell viability}

Lens epithelial cells remained viable and proliferated in the presence of all the concentrations of Ilomastat as detected by the WST-I assay. No significant differences between control
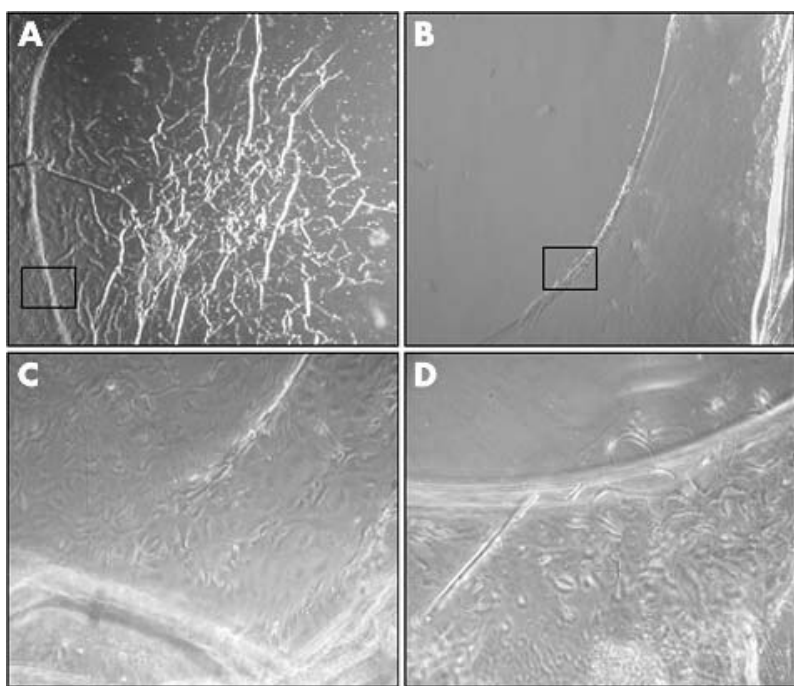

Figure 3 (A) Negative control treated lens capsule at day 15 demonstrating typical capsule wrinkling. The anterior capsule had also adhered onto the posterior capsule, which is not visible in the photograph. (B) Lens capsule that has been treated with $100 \mu \mathrm{M}$ llomastat at day 15. The capsule remains round with minimal capsule wrinkling compared with (A). At higher magnification of the boxed area of (A), the lens epithelial cells have clearly migrated over the posterior capsule to form a confluent layer (C). This contrasts with the marked area of (B), which shows virtually no lens epithelial cell migration onto the posterior capsule (D). (A and B, $\times 2.5$ magnification; $C$ and $D, \times 10$ magnification.)

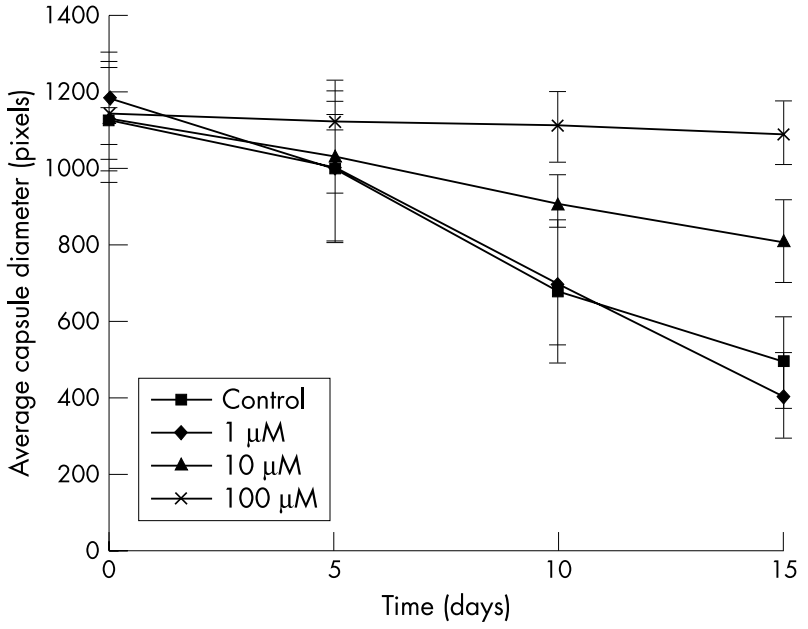

Figure 4 Cultured lens capsules were photographed at regular intervals and the average capsule diameters were digitally measured. The graph illustrates the effect of llomastat on capsule contraction. MMP inhibition resulted in a decrease in capsule contraction and therefore capsule diameter compared with control. Error bars indicating 95\% confidence interval have been included but fall within the data points.

and Ilomastat $(1 \mu \mathrm{M}, 10 \mu \mathrm{M}$, and $100 \mu \mathrm{M})$ treatments were found (fig 5).

\section{DISCUSSION}

Scar contracture plays an important role in the pathogenesis and failure of treatment of many ocular conditions, which include glaucoma, cataract (capsule contraction), trachoma, proliferative vitreoretinopathy, and diabetic retinopathy. Cell migration through the extracellular matrix along with collagen synthesis and deposition and matrix contraction are the key components to the scarring response. The data presented provide evidence to support the premise that MMP inhibition can reduce lens epithelial cell migration contraction of the capsule bag.

In response to surgery a wound healing response is triggered, and one of the key processes of healing is cell mediated contraction of the extracellular matrix. Residual lens epithelial cells are stimulated to proliferate and migrate onto the lens capsule by circulating growth factors and inflammatory cytokines. ${ }^{19} 20$ Cell migration is intrinsically involved with matrix contraction and previous reports have shown a reduction in cell migration and matrix contraction by inhibiting MMP activity. ${ }^{17}{ }^{21}$ The use of MMP inhibitors to modulate the scarring response in other ocular structures has

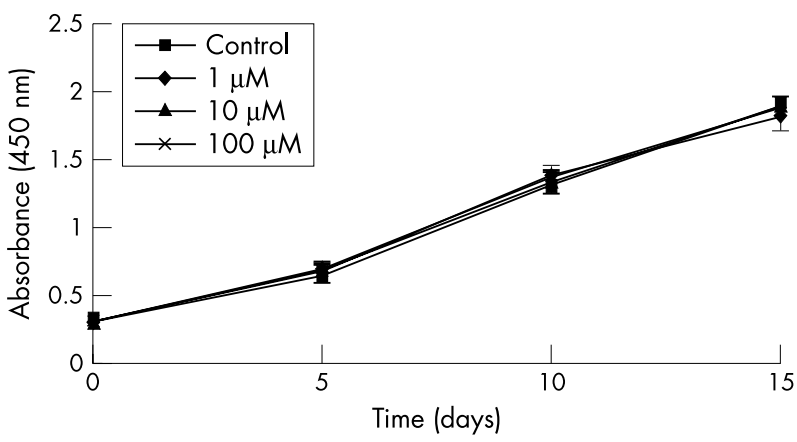

Figure 5 Viable LEC number measured with the WST assay. Cell proliferation in the presence of negative control, $1 \mu \mathrm{M}, 10 \mu \mathrm{M}$, and $100 \mu \mathrm{M}$ Ilomastat was quantified. No significant difference was found between proliferation rates in the different treatment groups. Error bars represent standard error of the mean. 
already been shown. Ozerdem et al have shown that progression of PVR indicated by scar tissue contraction can be significantly delayed with repeated intravitreal injections of the MMP inhibitor, prinomastat (AG3340). ${ }^{15}$ Furthermore, the level of MMP activity in the vitreous has been shown to be related to the development of retinal scarring, ${ }^{14}$ In addition, we recently reported a reduction in postoperative subconjunctival scarring associated with prolonged bleb survival following experimental glaucoma filtration surgery upon treatment with Ilomastat. ${ }^{22}$

In this culture model of PCO, $10 \%$ serum was used to accelerate the cellular processes that would normally occur at a slower rate in the human eye following cataract surgery. We report a decrease in MMP protein production with Ilomastat treatment leading to a reduction in cell migration and capsular bag contraction. MMP-2 and MMP-9 production were identified. Other MMP members have also been reported in the lens and their possible role in the development of PCO has yet to be determined. ${ }^{13}{ }^{23-25}$

A link between fibroblast migration and wound contraction has been proposed by Harris, who demonstrated that fibroblasts seeded onto a thin film of heat polymerised silicone oil wrinkle the substrate when the cells attempt to migrate. ${ }^{26}$ However, the cells do not move; the surface wrinkles. Harris proposed that the wrinkling was caused by cell tractional forces. This study provides further evidence of a possible link between migration and contraction as both process were inhibited to a similar degree by Ilomastat.

In this study we investigated whether MMP inhibition would reduce LEC migration. Cell migration onto the posterior capsule was dose dependently reduced with MMP inhibition. The magnification employed to record migration allowed clear identification of the LECs but limited measurement of the rate of cell locomotion to the first 7 days. A decrease in capsule contraction was observed alongside reduced cell migration with Ilomastat. By 7 days, prominent wrinkles and folds began to appear on the capsule. However, we found it difficult to accurately measure the actual distance travelled by the cells beyond this time due to capsule irregularity.

Several reports provide evidence that MMPs are involved in cell motility and contraction. Migrating fibroblasts are known to secrete collagenase, ${ }^{27}$ and with the addition of tissue inhibitor of metalloproteinases, fibroblast mediated collagen gel contraction decreases. ${ }^{28}$ Bullard et al reported impaired contraction in vitro by fibroblasts harvested from stromelysin-1 knockout mice compared with wild type. ${ }^{29}$ In addition, Scott et al demonstrated a reduction in collagen gel contraction as a direct result of inhibiting cell migration. The authors found that these inhibitory effects were reversed upon removal of the MMP inhibitor, suggesting that exposure to the agent does not lead to cellular toxicity. ${ }^{21}$ The result from the cell proliferation assay we performed is in agreement with this observation, and has also been recently reported in our laboratory. ${ }^{17}$

The anterior chamber aqueous flare and cell reaction that develops from a breakdown in the blood-aqueous barrier has been reported to subside after one month of surgery. ${ }^{30}$ Thus we hypothesise that MMP inhibition in the early stage following surgery may be all that is required to inhibit a significant amount of PCO development. Depending on the persistence of the MMP inhibitor in the lens bag, a slow release form may or may not be required. Possible slow release delivery systems are currently being investigated in our laboratory.

The molecular mechanisms that unite cell migration and wound contraction are not fully understood. As MMPs are capable of cleaving most matrix components, it is possible they play a role in integrin processing, modification of focal attachment to the underlying matrix and cell locomotion. It remains to be determined which MMPs are responsible for cleavage of lens epithelial cell focal adhesion sites and whether inhibiting their activity reduces migration on the capsule.

Matrix contraction plays a role in the pathogenesis and failure of treatment of most visually disabling conditions. As such, our data suggest a key role for the modulation of MMP activity in the inhibition of matrix contraction. MMP inhibition resulted in a profound reduction in lens epithelial cell migration and subsequent capsule contraction. Our findings provide further evidence that MMP inhibition prevents cell migration and capsule bag contraction, potentially making it suitable for inhibiting PCO.

\section{Authors' affiliations}

T T L Wong, J T Daniels, J G Crowston, P T Khaw, Wound Healing Research Unit, Department of Pathology, Institute of Ophthalmology, London, UK

T T L Wong, J G Crowston, P T Khaw, Moorfields Eye Hospital, London, UK

This work has been funded by the Wellcome Trust, UK (Grant number 060134; TLW), Royal National Institute for the Blind, UK (JTD), the Medical Research Council, UK (G9330070; PTK), and the Haymans Trust, London, UK.

\section{REFERENCES}

1 Nishi O, Nishi K, Wickstrom K. Preventing lens epithelial cell migration using intraocular lenses with sharp rectangular edges. J Cataract Refract Surg 2000;26:1543-9.

2 Nishi O, Nishi K, Akura J, et al. Effect of round-edged acrylic intraocular lenses on preventing posterior capsule opacification. J Cataract Refract Surg 2001;27:608-13.

3 Apple DJ, Solomon KD, Tetz MR, et al. Posterior capsule opacification. Surv Ophthalmol 1992;37:73-116.

4 Kappelhof JP, Vrensen GF. The pathology of after-cataract. A minireview. Acta Ophthalmol Suppl 1992;205:13-24.

5 Apple DJ, Peng Q, Visessook N, et al. Eradication of PCO: documentation of a marked reduction in $\mathrm{Nd}$ :YAG laser posterior capsulotomy rates in an analysis of 5416 pseudophakic human eyes obtained postmortem. Ophthalmology 2001;108:505-18.

6 Marcantonio JM, Rakic JM, Vrensen GF, et al. Lens cell populations studied in human donor capsular bags with implanted intraocular lenses. Invest Ophthalmol Vis Sci 2000;41:1130-41.

7 Saxby L, Rosen E, Boulton M. Lens epithelial cell proliferation, migration, and metaplasia following capsulorhexis. Br J Ophthalmol 1998;82:945-52.

8 Wormstone IM, Liu CS, Rakic JM, et al. Human lens epithelial cell proliferation in a protein-free medium. Invest Ophthalmol Vis Sci 1997;38:396-404.

9 Wong $\Pi$, Sethi C, Daniels JT, et al. Matrix metalloproteinases in disease and repair processes in the anterior segment. Surv Ophthalmol 2002;47:239-56.

10 Ishiguro N, Ito T, Miyazaki K, et al. Matrix metalloproteinases, tissue inhibitors of metalloproteinases, and glycosaminoglycans in synovial fluid from patients with rheumatoid arthritis. J Rheumatol 1999;26:34-40.

11 Westermarck J, Kahari VM. Regulation of matrix metalloproteinase expression in tumor invasion. Faseb J 1999;13:781-92.

12 Nwomeh BC, Liang HX, Cohen IK, et al. MMP-8 is the predominant collagenase in healing wounds and nonhealing ulcers. J Surg Res 1999;81:189-95

13 Kawashima Y, Saika S, Yamanaka O, et al. Immunolocalization of matrix metalloproteinases and tissue inhibitors of metalloproteinases in human subconjunctival tissues. Curr Eye Res 1998;17:445-51.

14 Kon CH, Occleston NL, Charteris D, et al. A prospective study of matrix metalloproteinases in proliferative vitreoretinopathy. Invest Ophthalmol Vis Sci 1998;39:1524-9.

15 Ozerdem U, Mach-Hofacre B, Cheng L, et al. The effect of prinomastat (AG3340), a potent inhibitor of matrix metalloproteinases, on a subacute model of proliferative vitreoretinopathy. Curr Eye Res 2000;20:447-53.

16 Fini ME, Cui TY, Mouldovan A, et al. An inhibitor of the matrix metalloproteinase synthesized by rabbit corneal epithelium. Invest Ophthalmol Vis Sci 1991;32:2997-3001.

17 Daniels JT, Cambrey AD, Occleston NL, et al. Matrix metalloproteinase inhibition modulates fibroblast-mediated matrix contraction and collagen production in vitro. Invest Ophthalmol Vis Sci 2003;44:1104-10.

18 Liu CS, Wormstone IM, Duncan G, et al. A study of human lens cell growth in vitro. A model for posterior capsule opacification. Invest Ophthalmol Vis Sci 1996;37:906-14.

19 Meacock WR, Spalton DJ, Stanford MR. Role of cytokines in the pathogenesis of posterior capsule opacification. Br J Ophthalmol 2000;84:332-6.

20 Kurosaka D, Nagamoto T. Inhibitory effect of TGF-beta 2 in human aqueous humour on bovine lens epithelial cell proliferation. Invest Ophthalmol Vis Sci 1994;35:3408-12. 
21 Scott KA Wood EJ, Karran EH. A matrix metalloproteinase inhibitor which prevents fibroblast-mediated collagen lattice contraction. FEBS Lett 1998;441:137-40.

22 Wong TT, Mead AL, Khaw PT. Matrix metalloproteinase inhibition modulates postoperative scarring after experimental glaucoma filtration surgery. Invest Ophthalmol Vis Sci 2003;44:1097-103.

23 Kawashima Y, Saika S, Miyamoto T, et al. Matrix metalloproteinases and tissue inhibitors of metalloproteinases of fibrous humans lens capsules with intraocular lenses. Curr Eye Res 2000;21:962-7.

24 Tamiya S, Wormstone IM, Marcantonio JM, et al. Induction of matrix metalloproteinases 2 and 9 following stress to the lens. Exp Eye Res 2000;71:591-7.

25 Smine A, Plantner JJ. Membrane type-1 matrix metalloproteinase in human ocular tissue. Curr Eye Res 1997;16:925-9.
26 Harris AK, Stopak D, Wild P. Fibroblast traction as a mechanism for collagen morphogenesis. Nature 1981;290:249-51.

27 Lambert CA, Soudant EP, Nusgens BV, et al. Pre-translational regulation of extracellular matrix molecules and collagenase expression in fibroblasts by mechanical forces. Lab Invest 1992:66:444-51.

28 Khaw PT, Occleston NL, Schultz G, et al. Activation and suppression of fibroblast function. Eye 1992;8:188-95.

29 Bullard KM, Mudgett J, Scheuenstuhl H, et al. Stromelysin-1-deficient fibroblasts display impaired contraction in vitro. J Surg Res 1999:84:31-4.

30 Pande M, Shah SM, Spalton DJ. Correlations between aqueous flare and cells and lens surface cytology in eyes with poly(methyl methacrylate) and heparinsurface-modified intraocular lenses. J Cataract Refract Surg 1995;21:326-30.

\section{$\mathrm{ECHO}$}

Isolation of a novel strain of adenovirus in epidemic keratoconjunctivitis

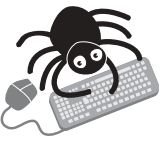

Please visit the British Journal of Ophthalmology website [www. bjophthalmol. com] for a link to the full text of this article.
A

novel strain of adenovirus has been isolated from a 25 year old male patient in Japan suffering from severe clinical manifestations of epidemic keratoconjunctivitis (EKC). adenoviruses: type 11 (Ad11) and type 35 (Ad35).

Although Adll infrequently causes keratoconjunctivitis, Ad35 and the new strain M86, have never before been reported as ocular pathogens. Analysis of M86 at a molecular level using restriction endonuclease showed that it might have evolved from the recombination of the two parent viruses Ad35 and Adll.

The fibre knob of M86, used to attach the virus to a specific cellular receptor on conjunctival or corneal cells, was found to have 99\% homology with Ad11. The hexon protein of M86, the antigen against which antibodies are raised to neutralise the infectivity of adenoviruses, was found to be partly homologous to Ad35 and partly to Adl1; the hypervariable regions (HVRs) of the hexon protein were 100\% homologous to Ad35 in HVRs $1,2,3$, and 6 and to Ad 11 in HVRs 4 and 6.

The mixed antigenic characteristics of a virus strain like M86 may enable it to circumvent existing immunity. This is important since it can enable the non-ocular adenoviruses to become ocular pathogens, which in the future might be responsible for outbreaks of EKC. 